\title{
Artículos
}

Encuentro №. 104, 6-28, 2016

\section{Ganadería lechera y deforestación en Nicaragua}

\author{
Juan Carlos Polvorosa* y Johan Bastiaensen* *
}

Recibido: junio de 2016 / Aceptado: julio de 2016

El dinamismo y el crecimiento de la producción y exportación de lácteos en Nicaragua han creado expectativas de crecimiento incluyente entre productores ganaderos lecheros, así como la reducción del avance de la frontera agrícola en el país, a través de la intensificación de la producción. Pero el modelo de crecimiento está sesgado en contra de pequeños y medianos productores pobres, quienes no logran tener acceso y aprovechar los beneficios económicos generados por el sector. Aquellos conectados a la producción y exportación de lácteos optan por crecer a través de la incorporación de más tierra a la producción bajo una lógica extensiva. Los productores excluidos aprovechan diferenciales de precio entre la vieja y nueva frontera agrícola para vender en la primera y migrar a la segunda donde pueden comprar más tierra a menor precio. El resultado es el continuo avance de la frontera agrícola sobre las últimas áreas de bosques tropicales de Nicaragua.

Palabras clave: lácteos, ganadería extensiva, deforestación, compra de tierra Clasificación JEL: O13, Q01, Q15

\section{Introducción}

La exportación de productos agropecuarios no-tradicionales ha tomado mucha importancia en Nicaragua, particularmente la de productos lácteos. Los lácteos han exhibido altos niveles de dinamismo en la economía, mostrando las más altas tasas de crecimiento porcentual en la producción de alimentos en el país en la última década

\footnotetext{
* Departamento de Economía Aplicada, Facultad de Ciencias Económicas y Empresariales, Universidad Centroamericana (UCA). Rotonda Rubén Darío 150 mts. al oeste, Apartado Postal 69, Managua, Nicaragua. Correo electrónico: jpolvorosa@ns.uca.edu.ni

** Institute of Development Policy and Management (IOB), Universidad de Amberes, Bélgica. Correo electrónico: johan.bastiaensen@uantwerpen.be
} 
(creciendo 164.17\% entre 2001 y 2011), así como el segundo mayor crecimiento porcentual del valor de exportaciones, de $471.72 \%$ para el mismo período (BCN, 2011). Este impresionante incremento es el resultado de una mayor demanda externa de lácteos, producto de los acuerdos de libre comercio firmados con Centroamérica y Estados Unidos. Al mismo tiempo, la ganadería lechera constituye una de las más importantes fuentes de ingresos para gran parte de la población rural de Nicaragua, especialmente para un segmento de pequeños y medianos productores con tierra ${ }^{1}$, representando el 63\% de las fincas lecheras del país (MAGFOR, 2008a; MAGFOR, 2005). En este contexto el apoyo al sector lechero, especialmente a los productores, ha sido una de las prioridades en las políticas de desarrollo rural de los últimos gobiernos a través de los Planes Nacionales de Desarrollo.

A su vez, dado el manejo tradicional extensivo de los sistemas de producción ganadera en el país, las políticas de apoyo al sector tienen el potencial de reducir la tasa de deforestación y avance de la frontera agrícola en Nicaragua. A través de la introducción de pastos mejorados sembrados, los productores experimentarían un aumento de la capacidad de carga animal de sus fincas (Sánchez, 2000), en la medida en que los pastos mejorados pueden soportar el doble de carga animal (UA/ $\mathrm{mz}^{2}$ ) que los pastos naturales. La intensificación de los sistemas productivos podría aumentar la producción de leche por $\mathrm{mz}$ y así disuadir la incorporación de más tierra a la producción e inclusive resultar en la liberación de área de pastos con el potencial de producir servicios ambientales, (MAGFOR, 2008a).

No obstante, la relación entre la demanda creciente de productos lácteos, la intensificación de la ganadería lechera y la reducción del avance de la frontera agrícola en Nicaragua, no ha sido totalmente estudiada o comprendida. Por el contrario, existe una idea equivocada que lleva a creer que la intensificación y la reducción de la deforestación pueden ser el resultado de un mayor dinamismo y precios de productos agropecuarios de alto valor agregado como los lácteos (MAGFOR, 2008a). La relación causa-efecto ha sido simplificada al asumir que mayores precios incentivarán a los productores a invertir en prácticas de producción más intensivas, en virtud de la adquisición de más tierra para la expansión de las actividades productivas. Algunas investigaciones resaltan que, en ciertas circunstancias, hacer la producción ganadera más productiva y/o rentable puede, contrario a lo esperado, poner más presión sobre las áreas de bosques tropicales (Kaimowitz \& Angelsen, 1998). Desde esta perspectiva, el análisis de las dinámicas locales del mercado de tierra donde la ganadería se desarrolla, debe ser incorporado al estudio de las decisiones de los productores de intensificar la producción al obtener mayores precios por sus productos. El análisis de los mercados de tierra puede enriquecer el entendimiento de la deforestación y el avance de la frontera agrícola en Nicaragua, en el contexto del acceso o exclusión de productores de los mercados más dinámicos y remunerativos de productos lácteos.

El objetivo de este artículo es revelar la dinámica que existe entre el boom de la producción láctea, mayores precios de la leche fresca y el concomitante problema

1 Un productor pequeño es definido como aquel con una finca menor a $50 \mathrm{mz}$ de tierra, uno mediano tiene entre 50 y $200 \mathrm{mz}$, y uno grande tiene más de $200 \mathrm{mz}$ de tierra (CENAGRO, 2001).

$2 \mathrm{UA}=400 \mathrm{~kg}$ de peso vivo. $\mathrm{Mz}=0.70$ hectáreas. 
de la migración interna y deforestación en Nicaragua. El artículo intenta dar cuenta de la reciente evolución de desarrollo de aquellos territorios en Nicaragua donde la ganadería lechera tiene lugar, incluyendo los productores, sus sistemas productivos y el grado de avance de la frontera agrícola y deforestación. El artículo presenta los resultados de un estudio de caso del territorio de Matiguás, un municipio ilustrativo del crecimiento y consolidación de la actividad lechera.

Matiguás es un territorio donde la ganadería lechera es la actividad económica dominante y donde, según el CENAGRO (2001), aproximadamente dos tercios de las fincas tienen ganado, principalmente pequeños productores (31.92\%) y medianos productores (58.90\%). Desde el año 2002 la expansión y mejora de la red vial de caminos secundarios y la disponibilidad de electricidad ha atraído y facilitado el establecimiento de empresas vinculadas a la producción, recolección y transformación de leche en productos lácteos de alto valor agregado. En 2011 operaban diez centros de acopio de leche con una capacidad combinada de recolección de 154 mil litros/ día, junto con dos plantas queseras semi-industriales con una capacidad combinada de procesamiento de $50 \mathrm{mil}$ litros/día. El incremento en la presencia de empresas ha resultado en una mayor demanda de leche fresca y precios más altos para los productores, lo que convierte a este municipio en un territorio ideal para un caso de estudio de la intensificación de la producción lechera y la evolución de la frontera agrícola.

Los resultados de esta investigación provienen de trabajo de campo realizado durante varios años en el municipio de Matiguás, culminando en 2011. El periodo de la investigación coincide con el establecimiento y operación de nuevos centros de acopio en el territorio entre 2003 y 2007 y la consolidación de la ganadería lechera como la principal actividad económica de Matiguás. La investigación permite valorar si se han producido cambios en los sistemas de producción ganaderos producto del boom de los lácteos y así evaluar el resultado sobre la deforestación en el territorio.

Los hallazgos y resultados aquí presentados provienen de la aplicación de métodos mixtos de investigación durante las fases recolección y análisis de datos. Durante las primeras fases de la investigación el territorio fue visitado en múltiples ocasiones, realizando investigación cualitativa exploratoria a través de entrevistas en profundidad entre 2007 y 2009, complementadas con la consulta de fuentes de información secundaria. Una serie de actores locales claves, incluyendo autoridades, empresas locales y personal de institutos de investigación y desarrollo en el territorio, fueron entrevistados para tener una mejor idea del mercado local de lácteos y un mayor conocimiento de las prácticas de producción ganadera lechera. Los resultados obtenidos de la fase cualitativa fueron utilizados como insumos en la generación de hipótesis de investigación. Durante una segunda fase, una encuesta representativa fue aplicada a 220 productores en diferentes zonas del territorio con el fin de corroborar los hallazgos cualitativos. Adicionalmente, para evaluar el desarrollo de la ganadería en Matiguás, también se usó datos de la CENAGRO 2001 para comparar con los resultados de la encuesta y describir las tendencias de evolución de los sistemas de producción ganaderos lecheros en diez años.

Este artículo se estructura en cinco secciones. La primera discute la relación que existe entre ganadería y deforestación, haciendo un repaso de los principales aportes teóricos escritos hasta ahora sobre el tema. La segunda sección analiza la 
dinámica de la ganadería lechera en Matiguás, a través de la descripción de las principales cadenas de valor, los precios de la leche y los factores de acceso y exclusión a la cadena de la leche fría pasteurizada. La sección tres aborda la evolución de los sistemas ganaderos lecheros, revisando los principales indicadores productivos de esta actividad. La cuarta sección discute el vínculo entre el boom lechero causado por los acopios de leche y el mercado de tierras de Matiguás, así como la distribución de la misma entre los productores.

\section{Ganadería y deforestación: marco teórico}

El avance de la frontera agrícola y la continua pérdida de bosques tropicales son vistas con gran preocupación dado sus efectos en la pérdida de biodiversidad, degradación de los suelos, contaminación y cambio climático (Dale, 1997; Fearnside, 2005; Steinfeld et al., 2006). Para poder detener o reducir la tasa de deforestación, es necesario comprender los factores que entran en juego y explican estos procesos. Geist y Lambin (2002) proveen un resumen comprensivo de la teoría sobre deforestación tropical alrededor del mundo, la cual es explicada por diversos factores que juegan a nivel próximo (como actividades humanas cercanas a los bosques) así como causas subyacentes que empujan la deforestación (procesos sociales a diversos niveles) que sostienen las causas a nivel próximo. Entre las primera los autores identifican la extensión de infraestructura, expansión de la agricultura y la extracción de madera; y entre las segundas mencionan factores demográficos, económicos, tecnológicos, culturales y políticos.

Kaimowitz (1996) analizó el desarrollo del sector ganadero en Centroamérica e identificó la construcción y extensión de infraestructura de transporte como uno de los principales factores que explican la conversión de áreas de bosques en pastizales. Adicionalmente, también identificó el papel jugado por la invasión de tierras públicas, la mejora en la seguridad de la tenencia, programas de colonización de tierra promovidos por el estado y las características inherentes de la actividad ganadera. A nivel próximo, Collins y Painter (1986) describen el proceso de colonización de tierras nuevas y deforestación en Centroamérica liderado por productores que emigran de otras zonas rurales hacia la frontera agrícola (Ilustración 1): el proceso de deforestación inicia con áreas boscosas de fácil acceso (producto de caminos para la explotación maderera), donde los inmigrantes limpian y cultivan la tierra para consumo del hogar y eventual venta; en la medida en que los suelos pierden fertilidad, los productores introducen pasturas y adoptan la ganadería. También, estos migrantes tienen la opción de vender su tierra a otro productor quien acumula y conforma grandes fincas explotadas bajo sistemas extensivos de ganadería (Nicholson et al., 1995). 


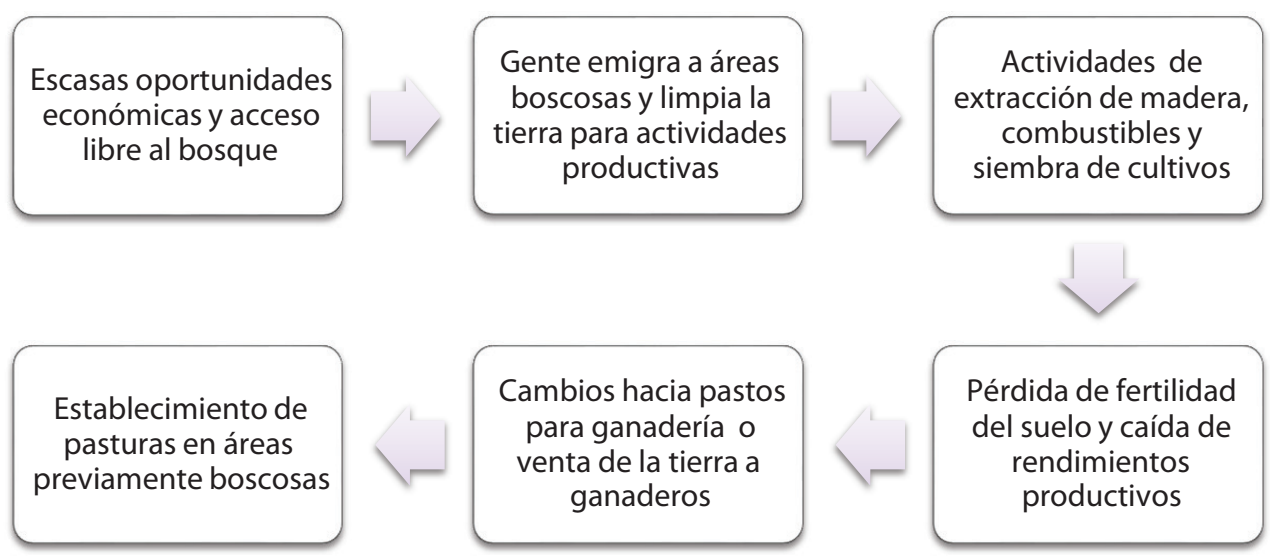

Ilustración 1. Proceso que lleva a la deforestación y sistemas de pastoreo Fuente: adaptado de Nicholson et al. (1995).

En Nicaragua, Maldidier (1993) identifica claros patrones de avance de la frontera agrícola en base a la colonización de áreas boscosas: la primera dinámica involucra la toma de tierras por grandes productores ganaderos y de café en zonas de bosque primario y secundario en la región central del país. Haciendo uso de colonos, estos productores fueron capaces de transformar estas áreas en pasturas y plantaciones permanentes. La segunda dinámica de avance de la frontera agrícola involucra a campesinos agricultores que a través de un proceso espontáneo de colonización de tierras altas y valles para la producción de café y granos, respectivamente, se asientan en nuevos territorios. La última dinámica es similar a la anterior, con la salvedad que estos productores no logran asentarse de manera permanente en las tierras en la medida en que son expulsados por grandes y medianos productores atraídos por el desarrollo de infraestructura de transporte de nuevos mercados. Bajo este contexto, el factor crítico es entender por qué los productores emigran hacia áreas boscosas y sirven de punta de lanza del continuo proceso de avance de la frontera agrícola que es, en última instancia, el factor subyacente que empuja la frontera agrícola y transforma áreas boscosas en pasturas para ganado.

Una de las principales causas identificadas y que empuja la deforestación es la presencia de oportunidades económicas inadecuadas, o la falta total de las mismas, para muchos productores (Collins \& Painter, 1986). La distribución desigual de tierra o su acceso bajo diferentes condiciones, la falta de acceso a tecnologías agropecuarias y oportunidades económicas remunerativas (como mercados y empleos) y la pobreza, constituyen factores que alienta la migración interna desde territorios de vieja frontera agrícola hacia áreas de bosque tropical (Mahar \& Schneider, 1994). Para Nicaragua, Maldidier y Antillón (1996) identifican que, en el contexto del desarrollo del modelo agro-exportador, la promoción y apoyo brindado por el estado únicamente a grandes empresas privadas conllevó al empobrecimiento de pequeños productores quienes, sin apoyo del estado, no pudieron aprovechar las oportunidades de mercado. Al ver las oportunidades de crecimiento bloqueadas, muchos productores pequeños y/o pobres eligen migrar y colonizar nuevos territorios. Esta migración representa una 
importante alternativa para los productores ubicados en la vieja frontera agrícola que buscan escapar un acelerado proceso de empobrecimiento.

Este patrón migratorio puede ser alentado por diferencias de precios de la tierra entre territorios. Mahar y Schneider (1994) reportan que productores con pequeñas parcelas ubicados en áreas con altos precios de la tierra enfrentan fuertes incentivos para vender su tierra y así comprar fincas más grandes en áreas donde la tierra sigue siendo barata. La diferencia de precio de la tierra entre territorios resulta de la cantidad de bosque disponible y el nivel de desarrollo del mercado de un territorio (White et al., 2001). Territorios con abundantes bosques y bajo desarrollo de mercados, como la frontera agrícola, resultan en precios más bajos de la tierra, en cambio, precios más altos tienen lugar en áreas con poca cobertura boscosa y accesibilidad a mercados, como territorios de vieja frontera agrícola. En un contexto así, es la falta de acceso a oportunidades económicas adecuadas que enfrentan pequeños y/o pobres productores, así como diferencias de precios de la tierra entre vieja y nueva frontera agrícola, lo que explica el proceso migratorio de productores hacia zonas boscosas y la deforestación.

En Nicaragua el movimiento migratorio desde comunidades del centro del país muestran un desplazamiento hacia el este (Costa Atlántica), y el avance de la frontera agrícola hacia los últimos territorios con presencia de bosque tropical. Mordt (2002) identificó que la principal presión del movimiento migratorio proviene de regiones interiores de Boaco, Chontales y Nueva Guinea, consideradas vieja frontera agrícola (donde la ganadería es la principal actividad productiva). Diferencias de precios de la tierra entre la región central más desarrollada y la frontera agrícola explican este proceso migratorio. De esta manera, pequeños y/o pobres productores de la vieja frontera agrícola (incluyendo Matiguás) venden su tierra para comprar nuevas y más grandes fincas en la nueva frontera agrícola donde la tierra sigue estando barata (Maldidier, 1993).

Comúnmente se considera que la solución para frenar o detener el avance de la frontera agrícola sobre los bosques tropicales es la adopción de prácticas productivas intensivas. Nicholson et al. (1995) definen un sistema de producción intensivo como aquel que provee mayor producción por animal y unidad de tierra en producción que aquel que se puede obtener a través de prácticas de producción tradicionales. Por ejemplo, el cambio de uso de pastos naturales por pastos mejorados sembrados y la adopción de prácticas silvo-pastoriles es considerado un avance importante hacia el uso sostenible de la tierra dado el aumento de los rendimientos productivos (Grau \& Aide, 2008; Yamamoto et al., 2007). Otros autores subrayan que, bajo varias circunstancias, hacer la ganadería más productiva y/o rentable, contrario a lo esperado, puede poner más presión sobre las áreas de bosque tropical (Kaimowitz $\&$ Angelsen, 1998). Aumentos de precios pueden elevar los retornos económicos de una actividad y de aquellos factores productivos vinculados a la misma, como la tierra, lo que puede alentar a los productores a incorporar más tierra a la producción resultando así en mayor deforestación (Griffith \& Zepeda, 1994; Nicholson et al., 1995); estos resultados pueden ser más pronunciados y graves en países donde existe disponibilidad y libre acceso a las áreas de bosques existentes (Tomich et al., 1998). En estas circunstancias, al aumentar los retornos económicos de la ganadería lechera, la incorporación de más tierra a la producción será el resultado más probable, en 
virtud de la adopción de prácticas productivas más intensivas en el área ya destinada a la producción.

White et al. (2001) enfatizan que es el precio de la tierra ex ante el principal determinante en la decisión de los productores de adoptar tecnologías productivas intensivas ${ }^{3}$ (como pastos mejorados); siempre que los precios de la tierra sean bajos los productores optarán por expandir la producción a través de la incorporación de más tierra, pero en la medida en que la tierra se encarezca y los productores no puedan comprar fácilmente más de este factor, elegirán incrementar la producción a través de tecnologías ligadas a la intensificación. Esto ha llevado a concluir que la escasez de bosque tropical es una pre-condición necesaria para que los productores adopten practicas intensivas de producción (Kaimowitz \& Angelsen, 2008; White et al., 2001).

Bajo este contexto la pérdida de bosque tropical en Centroamérica puede ser explicada, principalmente, por la dinámica creciente agro-exportadora que ha hecho de la ganadería lechera una actividad más rentable. A su vez, las barreras que excluyen a algunos productores de estas oportunidades (pequeños y/o pobres principalmente) empujan a los mismos a valorar posibilidades de migración hacia la nueva frontera agrícola, especialmente cuando existen altas diferencias en el precio de la tierra que hacen atractiva esta opción. Dado que aún existen en Centroamérica áreas boscosas desprotegidas y de libre acceso, los esfuerzos destinados a promover cambios productivos son obstaculizados y fracasan en detener el avance de la frontera agrícola en la medida en que persiste el sistema productivo basado en la compra y adición de más tierra a la producción (Tomich et al., 1998). A través de este proceso, los productores capaces de aprovechar las oportunidades económicas que ofrece la exportación de lácteos pasteurizados compran tierra de productores dispuestos a vender y reubicarse en la frontera agrícola, donde las tierra es más barata y pueden adquirir fincas más grandes (Humphries, 1998; Maldidier, 1993).

\section{Precios y mercado de la leche en Matiguás}

En esta sección tratamos el análisis del boom de la producción láctea en Matiguás basado en el incremento de la competencia entre los canales de comercialización de leche fresca que co-existen en el territorio. También analizamos el potencial que los diferentes canales tienen para incrementar los retornos de la producción ganadera lechera a través de precios más altos y estables, así como los factores que pueden bloquear el acceso de los productores a los mismos. La mayor parte del análisis aquí presentado proviene de información secundaria y datos de la encuesta aplicada a los productores en el territorio.

3 Otros factores también pueden jugar un rol importante: acceso a servicios financieros y no financieros, condiciones de los préstamos, limitaciones de mano de obra, entre otros, que explican la lenta adopción de tecnologías de intensificación (Bebe et al., 2003; Holmann, 1999). 


\subsection{Mercado de la leche en Matiguás}

En Matiguás existen tres canales de comercialización de la leche operando bajo cadenas de valor, los cuales producen, recolectan, comercializan y transforman la leche en productos lácteos: la cadena de la leche fría pasteurizada ligada a la gran industria pasteurizadora, la cadena semi-industrial de queso de exportación y la cadena del queso tradicional ${ }^{4}$. Cada cadena opera bajo diferentes modalidades de gobernanza que toman la forma de grados y estándares públicos y privados (implementados por una o varias empresas líderes) relacionados a la seguridad y calidad del producto a lo largo de la cadena. La estructura de gobernanza de la cadena se traduce en diferentes términos de intercambio (precios, cantidades, calidades) que conjuntamente determinan el acceso/exclusión de los productores a las distintas cadenas y los beneficios que puedan recibir de éstas. El cuadro 1 resume las principales características de cada una de las cadenas (para un análisis profundo de cada cadena, ver Polvorosa y Flores, 2014).

Cuadro 1. Características de las cadenas de valor lácteas

\begin{tabular}{|c|c|c|c|c|}
\hline Cadena de valor & $\begin{array}{l}\text { Orientación } \\
\text { de mercado y valor } \\
\text { agregado }\end{array}$ & $\begin{array}{l}\text { Gobernanza y } \\
\text { firma líder }\end{array}$ & $\begin{array}{l}\text { Beneficios para los } \\
\text { productores }\end{array}$ & $\begin{array}{l}\text { Términos } \\
\text { de acceso y } \\
\text { requisitos }\end{array}$ \\
\hline $\begin{array}{l}\text { Leche fría } \\
\text { pasteurizada }\end{array}$ & $\begin{array}{l}\text { *Para el mercado formal, } \\
\text { doméstico y externo. } \\
\text { *Alto nivel de valor } \\
\text { agregado. } \\
\text { *Variedad de productos } \\
\text { lácteos }\end{array}$ & $\begin{array}{l}\text { *Industria pasteurizadora } \\
\text { y centros de acopio. } \\
\text { *Cercana y estricta } \\
\text { coordinación de } \\
\text { volumen, calidad y } \\
\text { precios. }\end{array}$ & $\begin{array}{l}\text { * Precios más altos y } \\
\text { estables en el año. } \\
\text { *Insumos baratos. } \\
\text { * Asistencia técnica. } \\
\text { *Apoyo de ONG de } \\
\text { desarrollo. }\end{array}$ & $\begin{array}{l}\text { *Costos de } \\
\text { afiliación. } \\
\text { *Mínimo nivel de } \\
\text { volumen y calidad. } \\
\text { *Producción más } \\
\text { estable en el año. } \\
\text { *Inversiones en } \\
\text { finca para mejorar } \\
\text { condiciones y } \\
\text { equipamiento. }\end{array}$ \\
\hline $\begin{array}{l}\text { Queso semi- } \\
\text { industrial de } \\
\text { exportación }\end{array}$ & $\begin{array}{l}\text { *Mercado externo formal } \\
\text { e informal, (Centroamérica } \\
\text { y Estados Unidos.) *Nivel } \\
\text { medio de valor agregado. } \\
\text { * Principalmente queso } \\
\text { morolique. }\end{array}$ & $\begin{array}{l}\text { *Plantas de } \\
\text { procesamiento y } \\
\text { distribuidores mayoristas. } \\
{ }^{*} \text { Coordinación de } \\
\text { volumen, entrega y } \\
\text { calidad. }\end{array}$ & $\begin{array}{l}\text { *Altos precios al inicio } \\
\text { del año. } \\
\text { *Se compra todo el } \\
\text { excedente producido. } \\
\text { *Disponibilidad de } \\
\text { crédito inmediato para } \\
\text { emergencias. }\end{array}$ & $\begin{array}{l}\text { *Libre acceso. } \\
\text { *Algunos } \\
\text { requisitos de } \\
\text { calidad. }\end{array}$ \\
\hline Queso tradicional & $\begin{array}{l}\text { *Mercado doméstico } \\
\text { informal. } \\
\text { *Bajo nivel de valor } \\
\text { agregado. }\end{array}$ & $\begin{array}{l}\text { *No hay empresas } \\
\text { líderes. } \\
\text { *No existe } \\
\text { coordinación. } \\
\text { *Transacciones de } \\
\text { mercado. }\end{array}$ & $\begin{array}{l}\text { *Altos precios al inicio } \\
\text { del año. } \\
{ }^{*} \text { Compran toda la } \\
\text { leche que pueden sin } \\
\text { exigencia de calidad. }\end{array}$ & $\begin{array}{l}\text { *Libre acceso. } \\
\text { *Sin requisitos de } \\
\text { calidad y volumen. }\end{array}$ \\
\hline
\end{tabular}

Fuente: elaboración propia.

4 Adicionalmente operan intermediarios comerciales (transportistas) quienes juegan el rol de recolectar leche de los productores que luego es suplida a las tres diferentes cadenas de valor de lácteos. 
La cadena del queso tradicional ha estado presente todo el tiempo en el territorio y opera sin cuido ni reparo de los más básicos estándares de higiene y calidad públicos, lo que permite un acceso libre a todo tipo de productores. Sin embargo, las cantidades y precios del queso producido están sujetos a marcadas variaciones estacionales entre la temporada seca y la lluviosa, lo cual se traduce en precios altos durante la primera y bajos durante la segunda para todos los actores en los eslabones de la cadena. Esta condición hace de la cadena del queso tradicional la menos remunerativa y capaz de incrementar de manera sostenida los retornos de la ganadería lechera en Matiguás. No obstante, es el principal canal de comercialización para todos aquellos productores excluidos de las otras dos cadenas.

Por otro lado, la implementación de estándares públicos de calidad y seguridad a lo largo de la cadena semi-industrial del queso de exportación, hacen de ésta una cadena con mayores restricciones de acceso que la anterior. De manera muy similar también la cadena del queso semi-industrial de exportación experimenta variaciones estacionales en los precios del queso y la leche, lo que la hace menos interesante y remunerativa para los productores de leche. No obstante, pequeños grupos de grandes productores han logrado negociar precios más altos y estables en base a su capacidad de producir y proveer altos volúmenes de leche a lo largo del año.

La cadena de la leche fría pasteurizada ofrece el mayor potencial para incrementar los retornos económicos de la producción de leche de los productores, dado que paga los precios más altos y estables durante todo el año. Sin embargo, su acceso es limitado a pocos productores. Importantes barreras de acceso existen que evitan que productores pobres y/o pequeños suplan a la cadena de leche fría pasteurizada a través de los centros de acopio operando en el territorio; costos de membresía y niveles mínimos de producción son, ex-ante, requeridos a los productores que desean vender a los mismos. Adicionalmente, los productores deben cumplir con altos estándares de higiene y calidad que requieren de costosas inversiones en finca en equipos e infraestructura, lo que limita el acceso de los productores a esta cadena. En general, estas barreras actúan en contra de muchos productores excluyéndoles de la cadena de la leche fría pasteurizada y los beneficios que ésta ofrece vía precios e insumos.

\subsection{Precios de la leche en Matiguás}

Los datos de nuestra encuesta revelan que los productores que suplen a la cadena de leche fría pasteurizada se benefician de precios más altos y estables durante todo el año, en comparación con aquellos pagados por las otras dos cadenas de valor ${ }^{5}$. Los datos, al momento de la encuesta, muestran que los productores que suplen a los acopios reciben un precio promedio de $6.67 \mathrm{C} \$$ por litro (US $\$ 0.304 /$ litro $^{6}$ ) superior y estadísticamente significativo en comparación a los pagados por los competidores. Adicionalmente, la cadena de leche fría pasteurizada también paga precios altos durante la temporada seca y lluviosa, estadísticamente diferentes a los pagados

5 Se recogió precios del día de la encuesta, el precio más alto del verano y el precio más bajo del invierno.

61 US\$ = $21.9294 \mathrm{C} \$$ al momento de la encuesta. 
por la cadena del queso tradicional y los intermediarios comerciales. En relación a la estabilidad de precios durante el año, la cadena de leche fría pasteurizada muestra la menor reducción de precios (20.06\%) entre ambas temporadas del año, en comparación con los otros compradores, haciendo de esta cadena la más remunerativa para los productores.

Cuadro 2. Precios de la leche por compradores y temporada (C\$/litro)

\begin{tabular}{|c|c|c|c|c|c|}
\hline Época del Año & & $\begin{array}{c}1 \\
\text { Quesero } \\
\text { Tradicional }\end{array}$ & $\begin{array}{c}2 \\
\text { Intermediario } \\
\text { Comercial }\end{array}$ & $\begin{array}{c}3 \\
\text { Queso Semi- } \\
\text { industrial }\end{array}$ & $\begin{array}{c}4 \\
\text { Leche Fría } \\
\text { Pasteurizada }\end{array}$ \\
\hline \multirow{3}{*}{$\begin{array}{l}\text { Precio Actual } \\
\text { (Enero, 2011) }\end{array}$} & Media & 6.10 & 6.16 & 6.30 & 6.67 \\
\hline & Desviación St. & 0.65 & 0.38 & 0.49 & 0.42 \\
\hline & Diferente a & 4 & 4 & 4 & todos \\
\hline \multirow{3}{*}{$\begin{array}{l}\text { Precio del Verano } \\
(2010)\end{array}$} & Media & 6.38 & 6.37 & 6.55 & 6.78 \\
\hline & Desviación St. & 0.84 & 0.59 & 0.51 & 0.58 \\
\hline & Diferente a & 4 & 4 & & 1,2 \\
\hline \multirow{3}{*}{$\begin{array}{l}\text { Precio del } \\
\text { Invierno (2010) }\end{array}$} & Media & 4.24 & 3.94 & 4.94 & 5.42 \\
\hline & Desviación St. & 1.22 & 1.08 & 1.05 & 0.91 \\
\hline & Diferente a & 3,4 & 3,4 & 1,2 & 1,2 \\
\hline \multicolumn{2}{|c|}{$\begin{array}{l}\text { Diferencia porcentual entre } \\
\text { temporada seca y húmeda }\end{array}$} & -33.54 & -38.15 & -24.58 & -20.06 \\
\hline
\end{tabular}

Fuente: elaboración propia en base a datos de encuesta.

Por el contrario, la cadena del queso tradicional muestra precios bajos y sumamente inestables durante el año. En esta cadena, las cantidades y precios de la leche dependen de las fuerzas de la oferta y demanda de queso en Managua que a su vez dependen de la producción y oferta nacional de leche por temporadas. Durante la temporada seca de verano, la baja producción de leche para la producción de queso empuja los precios de éste, incrementando a su vez los precios pagados a los productores por la leche fresca. Al contrario, durante la temporada lluviosa la sobreproducción de leche reduce el precio del queso y a su vez los precios pagados a los productores en los territorios dedicados a la ganadería lechera. Los datos de nuestra encuesta (cuadro 2) confirman la variación de precios entre temporadas $(-33.54 \%)$. Adicionalmente, los precios pagados por la cadena de queso tradicional son los más bajos entre las tres cadenas (C\$6.10 o US\$0.27 por litro), haciendo de esta cadena la menos remunerativa para los productores de leche en Matiguás.

Información adicional de los precios de la leche, su estabilidad y evolución en el tiempo confirman la ventaja de vender a la cadena de la leche fría pasteurizada. En promedio, dicha cadena siempre paga precios más altos que aquellos pagados por las otras cadenas (Galetto \& Berra, 2011), y muestra menos variaciones porcentuales semanales de precios (MAGFOR, 2010). Esto confirma el potencial remunerativo de esta cadena para incrementar los retornos de la ganadería lechera entre productores de Matiguás. 


\subsection{Acceso y exclusión a la cadena de la leche fría pasteurizada}

A pesar del potencial beneficio de mayores y estables precios ofrecidos por la cadena de la leche fría pasteurizada, el acceso a dicha cadena es altamente restrictivo. Polvorosa (2013) identificó diversos factores claves que limitan el acceso de los productores a los centros de acopio en Matiguás. El primero y más importante es la disponibilidad de una ruta de recolección de leche operando por el camino más cercano a la finca del productor. Dado el carácter altamente perecedero de la leche cruda y la falta de equipos de enfriamiento en fincas, la disponibilidad de transporte hacia centros de acopio es clave para evitar el deterioro de la leche.

Un segundo aspecto lo conforman las características del sistema de producción implementado por los productores. La disponibilidad de suficiente pasto mejorado es una pre-condición para incrementar y estabilizar la producción de leche a lo largo del año. Adicionalmente, la disponibilidad de suficiente mano de obra es clave para el manejo del ganado y la producción lechera, especialmente durante las actividades de ordeño ${ }^{7}$ cuido del ganado. Asimismo, la disponibilidad de suficiente ganado propio en la finca para aprovechar la capacidad de carga de ésta y producir más leche (al menos 40litros ${ }^{8}$ ) para superar los costos de membrecía y transporte, son condiciones necesarias para poder vender a la cadena de la leche fría pasteurizada. También, la adopción de prácticas mejoradas durante el ordeño y manejo de la leche en base al uso de utensilio metálicos, mejoras en el área de ordeño y practicas higiénicas durante la extracción de la leche, son requeridas para cumplir con los grados y estándares establecidos e implementados a lo largo de la cadena de leche fría pasteurizada.

Un factor subyacente que explica la limitada introducción de pastos mejorados, disponibilidad de suficiente ganado y la adopción de prácticas mejoradas de ordeño es el acceso limitado que enfrentan los productores a créditos para inversión, lo que a su vez se traduce en la exclusión de muchos productores de los centros de acopio. Por último, el nivel de educación de los productores tiene un impacto positivo en los chances de los productores de suplir a la cadena de leche fría pasteurizada.

La combinación de estos factores actúa principalmente en contra de pequeños y medianos productores pobres, cuyos niveles de producción y/o baja calidad de la leche limita sus posibilidades de vender a la cadena de la leche fría pasteurizada. Por el contrario, la oportunidades económicas que ofrecen precios más altos y estables pagados por dicha cadena están siendo aprovechados por grandes y medianos productores con recursos, quienes cumplen con las condiciones requeridas por la cadena, limitando así el potencial causado por el boom de los lácteos a un grupo reducido de productores en Matiguás.

\section{Evolución de la ganadería en Matiguás}

En esta sección tratamos la evolución de la ganadería lechera, intentando identificar y analizar modificaciones vitales en los patrones de cambio de esta actividad en

En Nicaragua, el ordeño de la vaca se realiza de manera manual, casi exclusivamente.

8 Equivale a la cantidad que cabe en los depósitos utilizados para el transporte de la leche. 
el territorio durante la última década. Se discute en qué medida los productores de leche han intensificado sus sistemas productivos y si estos cambios se ven reflejados en mayores cargas animales en finca, así como mayores rendimientos productivos. Conjuntamente, estos cambios productivos confirmarían un proceso de especialización e intensificación a nivel de finca, y el progresivo abandono de sistemas productivos extensivos que tradicionalmente persisten en Nicaragua. La mayor parte del análisis aquí presentado proviene de la comparación de datos entre la CENAGRO 2001 y nuestra encuesta (2011). De esta manera podremos identificar el patrón de cambio de la ganadería lechera en Matiguás. Reconocemos, no obstante, que la comparación de datos entre una muestra representativa y el universo puede ser problemática (ver Polvorosa, 2013, para mayores detalles metodológicos).

\subsection{Apoyo a la reconversión ganadera y la intensificación}

Durante la última década la aparición de centros de acopio vinculados a la cadena de la leche fría ha aumentado la competencia y precios por la leche fresca en Matiguás, incrementando los retornos económicos de la ganadería lechera y consolidándola como la principal actividad económica del territorio. Adicionalmente, importantes cambios en los sistemas productivos ganaderos han sido fomentados y apoyados por organismos de investigación y desarrollo, así como instituciones (micro) financieras locales.

La re-conversión de los sistemas ganaderos lecheros ha sido apoyada a través de servicios financieros que han ampliado su cobertura en Matiguás, así como por servicios de asistencia técnica orientada hacia la intensificación de la producción. En el territorio, el Fondo de Desarrollo Agropecuario (FONDEAGRO) y Nitlapán son las dos principales instituciones que han sobresalido en el apoyo que han brindado a los productores. Dichas intervenciones tienen por convicción que la intensificación de la ganadería lechera tiene el potencial de crear oportunidades de inclusión de pequeños y medianos productores en la cadena de la leche fría pasteurizada, así como de promover prácticas productivas amigables con el medio ambiente.

Los esfuerzos de Nitlapan y FONDEAGRO han sido complementados por la provisión de servicios financieros a los productores destinados a la inversión en equipos e infraestructura en fincas. Al comparar los datos de la CENAGRO 2001 y el año $2008^{9}$ se puede ver que la cobertura crediticia se incrementó de $14.60 \%$ a $41.12 \%$ de los productores. Este incremento en el acceso a crédito se explica parcialmente por los convenios establecidos entre FONDEAGRO y micro-financieras locales para canalizar recursos a productores en Matiguás de cara a transformar y mejorar sus sistemas productivos. En el caso particular del Fondo de Desarrollo Local (FDL), por ejemplo, la institución diseñó un producto financiero llamado 'paquete verde', orientado hacia la intensificación de la producción lechera.

9 Utilizamos datos de 2008 para evitar el impacto de la crisis financiera internacional y nacional (que resultó del Movimiento No-pago) y mostrar la cobertura crediticia en Matiguás. Como resultado de ambas crisis, el nivel de recursos canalizados por las micro-financieras se redujo drásticamente, limitando la cobertura crediticia a productores de Matiguás y el resto del país desde 2008. 


\subsection{Evolución de los principales indicadores técnicos de producción}

En Matiguás la ganadería lechera se ha expandido y consolidado como principal actividad productiva del territorio. Al comparar los datos de uso de suelo en finca entre 2001 y 2011 es evidente que el territorio ha experimentado una expansión de la ganadería lechera a través de la orientación de mayores proporciones de tierra en fincas a ésta actividad (ver cuadro 3). Los datos revelan un aumento significativo del porcentaje de tierra de las fincas dedicado a la ganadería, pasando de $67.39 \%$ en 2001 a $82.57 \%$ en 2011. Esta expansión ha tenido lugar a expensas de la tierra previamente destinada a la agricultura y la colonización interna de tierra dentro de las fincas que antes estaba dedicada a áreas verdes. En el caso de la agricultura, no sólo se ha reducido el porcentaje de productores que destinaban tierra a esta actividad, de $89.12 \%$ a $82.71 \%$, también se ha reducido la proporción de tierra destinada a agricultura, de $17.55 \%$ a $11.51 \%$. De manera similar, a pesar del pequeño ascenso $(4.48 \%)$ de productores que reportan tener tierra de área verde ${ }^{10}$, el porcentaje total de la tierra destinada a este fin se ha reducido significativamente, de $21.41 \%$ a $9.80 \%$.

Cuadro 3. Uso de la tierra en fincas (2001-2011)

\begin{tabular}{|c|c|c|c|}
\hline Uso de la tierra en finca & Año & & \\
\hline \multirow{7}{*}{$\begin{array}{l}\text { Porcentaje de tierra de } \\
\text { la finca en ganadería }\end{array}$} & \multirow{3}{*}{2001} & $\%$ de productores & 100 \\
\hline & & Media & 67.39 \\
\hline & & Deviación St. & 23.65 \\
\hline & \multirow{3}{*}{2011} & $\%$ de productores & 100 \\
\hline & & Media & 82.57 \\
\hline & & Deviación St. & 13.18 \\
\hline & \multicolumn{2}{|c|}{ Evolución } & 15.18 \\
\hline \multirow{7}{*}{$\begin{array}{l}\text { Porcentaje de tierra de } \\
\text { la finca en agricultura }\end{array}$} & \multirow{3}{*}{2001} & $\%$ de productores & 89.12 \\
\hline & & Media & 17.55 \\
\hline & & Deviación St. & 15.36 \\
\hline & \multirow{3}{*}{2011} & $\%$ de productores & 82.71 \\
\hline & & Media & 11.51 \\
\hline & & Deviación St. & 8.59 \\
\hline & \multicolumn{2}{|c|}{ Evolución } & -6.04 \\
\hline \multirow{7}{*}{$\begin{array}{l}\text { Porcentaje de tierra } \\
\text { de la finca en área verde }\end{array}$} & \multirow{3}{*}{2001} & $\%$ de productores & 67.48 \\
\hline & & Media & 21.41 \\
\hline & & Deviación St. & 18.27 \\
\hline & \multirow{3}{*}{2011} & $\%$ de productores & 71.96 \\
\hline & & Media & 9.8 \\
\hline & & Deviación St. & 7.85 \\
\hline & \multicolumn{2}{|c|}{ Evolución } & $\mid-11.61$ \\
\hline
\end{tabular}

Fuente: elaboración propia en base a CENAGRO 2001 y datos de la encuesta.

10 Esto puede ser el resultado de proyectos implementados en el territorio por Nitlapán y otros organismos de desarrollo, que han incrementado la valoración positiva de los productores sobre las áreas verdes en finca, que previamente se consideraban sin valor alguno. 
La evolución de la ganadería lechera en Matiguás apunta hacia la expansión de esta actividad en el territorio y la consolidación del mismo como vieja frontera agrícola, basada en las características del territorio y de sus sistemas productivos. El territorio muestra la sustitución de sistemas productivos diversificados que combinan la agricultura con la ganadería (tradicionalmente implementados por pequeños productores), por uno más especializado en ganadería lechera. La expansión de la ganadería lechera ha supuesto el sacrificio y reducción de las áreas verdes internas del territorio, que han sido incorporadas a esta actividad. Ambos patrones de evolución sugieren la consolidación de la ganadería lechera como una de las principales actividades productivas del territorio. Adicionalmente muestran la persistencia del sistema productivo extensivo basado en la incorporación de más tierra para alcanzar crecimiento económico.

Los datos de evolución de la ganadería lechera en base a la proporción de pastos mejorados en la finca (del área destinada a la ganadería) y la capacidad de carga de ganado hembra, muestra un cambio algo paradójico en términos de intensificación. Los datos de presencia de pastos mejorados en finca aumentaron de $56.31 \%$ a $100.00 \%$ entre 2001 y 2011, pero la proporción reportada de cobertura dentro del área destinada a ganadería muestra una disminución de $49.00 \%$ a $36.38 \%$ para el mismo período. Antes de continuar con nuestro análisis es importante destacar los problemas que pueden existir en el nivel de precisión de la medición de la variable de pastos mejorados, tanto en la CENAGRO como en nuestra encuesta. A pesar de que ambos cuestionarios preguntan por la cantidad de tierra cubierta por pastos mejorados, los dos fallan en dar un tratamiento adecuado a las condiciones del pasto en cuestión ${ }^{11}$. En este sentido, creemos que los productores pueden haber reportado tener pastos mejorados en sus fincas a pesar de su escasa presencia y degradación en algunos potreros, conllevando a la sobreestimación de la proporción real de los mismos. Producto de la degradación de los pastos por sobrepastoreo y falta de mantenimiento, es común encontrar potreros con parches de pastos mejorados combinados con pastos naturales e inclusive maleza, y aun así pueden haber sido considerados como pastos mejorados.

El porcentaje de productores que reportan la presencia de pastos mejorados en sus fincas realmente ha aumentado en los últimos diez años y es consistente con los esfuerzos desarrollados en el territorio para promover la intensificación ganadera entre los productores. Por otro lado, la reducción de la proporción de la finca con pastos mejorados no es algo improbable y puede ser explicado por diversos factores. Primero, la incorporación de tierra adicionales, previamente destinadas a áreas verdes (y en menor medida a la agricultura) aumentó el área total en ganadería, lo cual pudo haber reducido la proporción inicial de pastos mejorados reportado por los productores. Segundo, los altos costos de establecimiento de pastos mejorados para pastoreo pueden también haber limitado su expansión y/o reposición dentro del área destinada a pastoreo. Tercero, también producto de sus altos costos de

11 Barrios (2002), por ejemplo, identificó que 15.4\% de los productores que entrevistó en 2002 en Matiguás tenían más del $50 \%$ de sus potreros compuestos de pasturas degradadas o habían perdido su cobertura forrajera. Dicho porcentaje subía al 25\% de los productores al finalizar la temporada seca de verano. 
establecimiento, los productores pudieron haber priorizado la introducción de bancos forrajeros (no aptos para pastoreo y utilizados principalmente en la época seca del verano), los cuales generalmente sólo cubren pequeñas áreas del total de la finca, reduciendo así su proporción dentro de toda el área destinada a ganadería.

A pesar de las limitaciones, creemos que la información recogida por ambos instrumentos puede ser aún de utilidad para establecer patrones de evolución de los sistemas ganaderos lecheros en el territorio. La alta presencia de pastos mejorados en las fincas y su baja cobertura sugieren que la ganadería lechera en Matiguás sigue un patrón de expansión extensivo basado en la incorporación de más tierra, en vez de uno intensivo basado en pastos mejorados que aumenten los rendimientos por unidad de tierra destinada a esta actividad.

Cuadro 4. Intensificación de la ganadería lechera (2001-2011)

\begin{tabular}{|c|c|c|c|}
\hline Indicador & Año & & \\
\hline \multirow{7}{*}{$\begin{array}{l}\text { Porcentaje de Cobertura de Pastos } \\
\text { Mejorados }\end{array}$} & \multirow{3}{*}{2001} & $\%$ de productores & 56.31 \\
\hline & & Media & 49 \\
\hline & & Deviación St. & 35.99 \\
\hline & \multirow{3}{*}{2011} & $\%$ de productores & 100 \\
\hline & & Media & 36.38 \\
\hline & & Deviación St. & 29.73 \\
\hline & \multicolumn{2}{|c|}{ Evolución } & -12.62 \\
\hline \multirow{7}{*}{ Carga Animal Hembra de la Finca (UA/mz) } & \multirow{3}{*}{2001} & $\%$ de productores & 98.31 \\
\hline & & Media & 0.57 \\
\hline & & Deviación St. & 0.45 \\
\hline & \multirow{3}{*}{2011} & $\%$ de productores & 100 \\
\hline & & Media & 0.73 \\
\hline & & Deviación St. & 0.33 \\
\hline & \multicolumn{2}{|c|}{ Evolución } & 0.17 \\
\hline
\end{tabular}

Fuente: elaboración propia en base a CENAGRO 2001 y datos de la encuesta.

Los datos también muestran que a pesar de la reducción de la proporción de tierra con pastos mejorados, las fincas han experimentado un aumento de su carga animal hembra, pasando de 0.57 a $0.73 \mathrm{UA} / \mathrm{mz}$, entre 2001 y 2011. Este incremento puede ser parcialmente atribuido a la re-orientación productiva del hato en finca, donde los productores han reducido el porcentaje de ganado macho para la producción de carne y lo han sustituido por ganado hembra para la producción de leche, resultando en sistemas productivos ganaderos orientados a la producción de leche, en vez del tradicional doble-propósito, incrementando la carga animal hembra en las fincas.

Por otro lado, los indicadores globales de producción de leche en Matiguás han alcanzado ciertas mejoras en términos de litros por vaca y por manzana de tierra bajo ganadería. En diez años el promedio general de litros/vaca/día ha 
aumentado de 3.861ts a 4.171ts (cuadro 5). Creemos que este relativo progreso es el resultado de procesos de intensificación parcial de los sistemas productivos basados en la adopción bancos forrajeros para alimentación, aunque solamente se utilicen durante la temporada seca de verano. A su vez, ambos aumentos, en la carga animal hembra de las fincas y la producción litros/vaca/día, son determinantes clave de la producción anual de leche por manzana ${ }^{12}$ de tierra bajo producción. Cuando analizamos este indicador productivo encontramos que el volumen anual de leche producida por manzana de tierra en ganadería ha aumentado 64.4lts en los últimos diez años.

Cuadro 5. Rendimientos de la ganadería lechera (2001-2011)

\begin{tabular}{|c|c|c|c|}
\hline Indicador (Its) & Año & & \\
\hline \multirow{7}{*}{ Producción por día por vaca } & \multirow{3}{*}{2001} & $\%$ de productores & 90.92 \\
\hline & & Media & 3.86 \\
\hline & & Deviación St. & 1.52 \\
\hline & \multirow{3}{*}{2011} & \% de productores & 100 \\
\hline & & Media & 4.17 \\
\hline & & Deviación St. & 1.21 \\
\hline & \multicolumn{2}{|c|}{ Evolución } & 0.31 \\
\hline \multirow{7}{*}{$\begin{array}{l}\text { Producción anual de leche por mz de } \\
\text { tierra en ganadería }\end{array}$} & \multirow{3}{*}{2001} & \% de productores & \begin{tabular}{|l|}
89.48 \\
\end{tabular} \\
\hline & & Media & 284.12 \\
\hline & & Deviación St. & 277.98 \\
\hline & \multirow{3}{*}{2011} & \% de productores & 100 \\
\hline & & Media & 352.52 \\
\hline & & Deviación St. & 232.14 \\
\hline & \multicolumn{2}{|c|}{ Evolución } & 68.4 \\
\hline
\end{tabular}

Fuente: Elaboración propia en base a CENAGRO 2001 y datos de la encuesta.

La evolución de los indicadores técnicos de producción vinculados a la ganadería lechera en Matiguás demuestran que el sector ha alcanzado progresos positivos, aunque esta evolución es menor de lo que se esperaba dado el dinamismo del mercado de leche local y el apoyo financiero y no financiero brindado al sector. Contrario a lo esperado, el boom lechero no ha llevado a un proceso decisivo y abrumador de intensificación de los sistemas ganaderos lecheros. La ganadería lechera se ha expandido pero principalmente en base a la incorporación de más tierra al sistema productivo, a expensas de tierra previamente utilizada en la agricultura y áreas verdes y la sustitución de ganado macho por hembra. La combinación de estos factores explica el avance pequeño, pero positivo, de los indicadores de

12 La producción anual de leche por manzana es calculada sobre el área de la finca destinada a la ganadería que está compuesta de pastos naturales y pastos mejorados (incluyendo los bancos forrajeros). 
rendimientos productivos. Estos hallazgos suponen la continuidad y persistencia de la lógica productiva extensiva tradicional basada en la incorporación de más tierra al sistema productivo, poniendo presión en las últimas áreas de bosque disponibles en Matiguás y otras zonas del país.

\section{Mercado de tierra en Matiguás}

En Matiguás, desde antaño tiene lugar un proceso natural y continuo de compra y venta de tierra. No obstante, desde el año 2005 y con la aparición de los centros de acopio, el mercado de tierra ha tomado mayor impulso. En este contexto, en esta sección exploramos la dinámica del mercado de tierra en Matiguás para evaluar en qué medida las ventas y compras de tierra en años recientes están vinculadas al boom lechero y a la migración interna de productores y el avance de la frontera agrícola. Con este objetivo en mente, en esta sección discutimos la dinámica reciente del mercado de tierra desde la aparición de los centros de acopios a través del historial de compra-venta de tierra ${ }^{13}$ entre productores del territorio a partir de los datos de nuestra encuesta.

\subsection{Evolución del mercado de tierra en Matiguás}

El análisis del mercado de tierra en Matiguás requiere revelar y entender cuáles son las principales fuerzas que sustentan la oferta y demanda de tierra y su relación con la dinámica de la leche en el territorio. Actualmente tres fenómenos pueden ser identificados detrás de la dinámica del mercado de tierra en Matiguás: i) divisiones de fincas para herencia, ii) ventas totales o parciales de fincas, y iii) compras y absorción de nuevas tierra por fincas ya existentes.

Las herencias de tierra a través de la división de una finca constituyen un mecanismo por medio del cual muchos productores inician sus actividades productivas propias. La herencia ocurre principalmente ante la muerte de un productor, quien divide y deja a sus descendientes porciones iguales de la finca original. En otros casos, cuando el productor tiene más de una finca, deja a cada uno de sus descendientes una de éstas. En algunas ocasiones el productor hereda en vida a sus hijos para proveerles de sus propios medios de vida a través de la producción en finca. El proceso involucra destinar (legalmente o de facto) un pedazo de la finca a cada hijo, donde éste puede desarrollar actividades productivas a la vez que el padre dueño sigue trabajando el resto de la tierra. Los datos de nuestra encuesta muestran que $27.7 \%$ de los productores entrevistados obtuvieron su finca a través de la herencia y $2.3 \%$ por medio de la herencia en vida, así como $5.45 \%$ de los productores entrevistados ya habían heredado parte de su finca a sus hijos.

13 A los productores entrevistados se les pidió recordar las últimas transacciones de venta de tierras que tuvieron lugar en su comunidad en los pasados cinco años, la cantidad de tierra vendida, si era parte o la totalidad de la finca y la razón que impulsó al productor a vender la misma. También se les pidió recordar las compras de tierra que ellos habían realizado adyacentes a su finca o de nuevas fincas, tanto dentro como fuera del territorio de Matiguás, recordando el año de la compra y la cantidad de tierra adquirida. 
Otra fuerza importante que impulsa el mercado local de tierra es la venta parcial o total de fincas en el territorio. El cuadro 6 muestra información sobre las ventas de tierra en los últimos seis años separada por la proporción de la finca vendida (un pedazo o todo) y las razones de la venta. Al analizar los datos desde 2005 encontramos que la mayoría de las transacciones de venta $(63.01 \%)$ involucraron la venta de toda la finca, mientras que la principal razón citada para la venta $(39.04 \%)$ fue la compra de más tierra. Cuando se analiza la razón que explica la venta, el pago de deuda explica el $56.14 \%$ de las ventas parciales (un pedazo), en cambio, la compra de (más) tierra explica la venta completa de fincas.

Cuadro 6. Proporción de tierra vendida y razones de la venta

\begin{tabular}{|c|c|c|c|c|c|c|}
\hline \multirow{2}{*}{$\begin{array}{l}\text { Proporción de la finca } \\
\text { vendida }\end{array}$} & \multicolumn{4}{|c|}{ Razones de la venta (porcentaje) } & \multirow{2}{*}{$\begin{array}{l}\text { Ventas } \\
\text { totales }\end{array}$} & \multirow{2}{*}{$\begin{array}{l}\text { Porcen- } \\
\text { taje }\end{array}$} \\
\hline & $\begin{array}{l}\text { Pago de } \\
\text { deuda }\end{array}$ & $\begin{array}{l}\text { Compra } \\
\text { de tierra }\end{array}$ & Otra & $\begin{array}{l}\text { No } \\
\text { sabe }\end{array}$ & & \\
\hline Un pedazo & 56.14 & 17.39 & 25.00 & 39.13 & 54 & 36.99 \\
\hline Toda & 43.86 & 82.61 & 75.00 & 60.87 & 92 & 63.01 \\
\hline TOTAL & 100.00 & 100.00 & 100.00 & 100.00 & 146 & 100.00 \\
\hline Porcentaje & 15.75 & 39.04 & 31.51 & 13.70 & 100.00 & \\
\hline
\end{tabular}

Fuente: Elaboración propia en base a datos de encuesta.

Una de las razones para las recientes ventas en el territorio está relacionada a la falta de suficiente liquidez de los productores para hacer frente al pago de préstamos contraídos vinculados a la compra de ganado, principalmente. Por un lado, la expansión de la cobertura crediticia de las instituciones financieras operando en Matiguás previo a 2008 llevo a una fuerte competencia entre las instituciones que apostaban por el mismo segmento de mercado, resultando en el sobreendeudamiento de muchos productores que tomaron más de un préstamo al a vez. Por otro lado, muchos productores que recibieron préstamos para financiar la compra de ganado enfrentaron dificultades en el pago de los mismos producto de la crisis del ganado que resultó en una caída del precio de la carne de un 50\% en 2008 (Pérez, 2011). Bajo este contexto, muchos productores resolvieron sus necesidades de liquidez a través de la venta de parte de sus tierras para evadir el embargo total de sus fincas puestas como garantía. En otros casos, algunos productores vendieron toda su finca para cancelar el préstamo y en el caso de obtener un sobrante, lo utilizaron para comprar una nueva finca más pequeña.

Alternativamente, la principal razón citada para las ventas de tierra en Matiguás en los últimos años está relacionada con la compra de tierra adicional afuera del territorio, presumiblemente en la frontera agrícola. Diferencias en los precios de la tierra han alentado a muchos productores a emigrar al este del país (Costa Atlántica principalmente), donde el precio de la tierra se mantiene bajo, para comprar mayores extensiones y aumentar la escala de producción. Los datos de la encuesta muestran que la mayoría de las ventas de fincas para comprar más tierra $(82.61 \%)$ involucraron la venta de toda la finca y la salida del productor del territorio para reubicarse en algún territorio cercano a la frontera agrícola para comprar más tierra de la que 
tenía inicialmente. Por medio de este proceso los productores pueden incrementar la escala de producción de sus fincas en base a la disponibilidad de tierra adicional, sin necesidad de intensificar sus sistemas productivos. Adicionalmente, los datos del tamaño promedio y desviación estándar de las fincas vendidas $(69.97 \mathrm{mz}$ y $72.74 \mathrm{mz})$ muestran que son pequeños y medianos productores principalmente los que venden su propiedad y emigran hacia la frontera agrícola.

De acuerdo al historial de adquisición de tierra de los productores entrevistados, las ventas de tierra han sido acompañadas por un proceso dinámico de compras tanto dentro como fuera de Matiguás. Los datos sugieren que las compras son un proceso natural vinculado al aumento de los sistemas productivos que buscan cómo crecer a través de la incorporación de más tierra a la producción en virtud de intensificar la producción con la misma cantidad de tierra de la finca. A pesar de que este proceso es natural en el territorio, el mismo se aceleró a partir de 2005, cuando los centros de acopio comenzaron a operar en Matiguás.

Para el análisis, las compras fueron separadas en dos tipos: tierra adyacente a la finca (ya sea un pedazo o la totalidad de la finca) comprada a un vecino para ser incorporada al sistema productivo de la finca del productor; y compras no adyacentes de tierra (en Matiguás o en otro sitio) que se convierte en una nueva finca o unidad productiva. Además, los datos de compra de tierra de los productores fueron separados por la cadena de valor a la cual suple el productor que adquiere la tierra. Éstos sugieren que las compras de tierra están vinculadas a la expansión de la ganadería lechera (bajo la lógica de añadir más tierra al sistema productivo) por parte de productores que mayoritariamente venden a la cadena de la leche fría pasteurizada. 
Cuadro 7. Compra de tierra desde 2005 por canal de comercialización

\begin{tabular}{|c|c|c|c|c|c|c|}
\hline $\begin{array}{c}\text { Tipo de } \\
\text { compra de } \\
\text { tierra }\end{array}$ & & $\begin{array}{c}\text { Quesero } \\
\text { tradicional }\end{array}$ & $\begin{array}{l}\text { Intermedia- } \\
\text { rio comercial }\end{array}$ & $\begin{array}{c}\text { Quesero } \\
\text { semi-industrial } \\
\text { de exportación }\end{array}$ & $\begin{array}{c}\text { Leche fría } \\
\text { pasteuri- } \\
\text { zada }\end{array}$ & TOTAL \\
\hline \multirow{5}{*}{$\begin{array}{l}\text { Adyacente } \\
\text { a la finca }\end{array}$} & $\begin{array}{l}\text { Porcentaje de } \\
\text { productores con } \\
\text { compra }\end{array}$ & 15.22 & 15.94 & 17.02 & 20.69 & 17.27 \\
\hline & $\begin{array}{l}\text { Numero de compras } \\
\text { realizadas }\end{array}$ & 9 & 11 & 9 & 15 & 44 \\
\hline & $\begin{array}{l}\text { Porcentaje de compras } \\
\text { realizadas }\end{array}$ & 20.45 & 25.00 & 20.45 & 34.09 & 100.00 \\
\hline & $\begin{array}{l}\text { Mz de tierra } \\
\text { compradas }\end{array}$ & 115.5 & 99 & 104 & 682 & 1000.5 \\
\hline & $\begin{array}{l}\text { Porcentaje de tierra } \\
\text { comprado }\end{array}$ & 11.54 & 9.90 & 10.39 & 68.17 & 100.00 \\
\hline \multirow{5}{*}{$\begin{array}{l}\text { Finca } \\
\text { adicional }\end{array}$} & $\begin{array}{l}\text { Porcentaje de } \\
\text { productores con } \\
\text { compra }\end{array}$ & 6.52 & 2.90 & 10.64 & 13.79 & 8.18 \\
\hline & $\begin{array}{l}\text { Numero de compras } \\
\text { realizadas }\end{array}$ & 4 & 2 & 5 & 9 & 20 \\
\hline & $\begin{array}{l}\text { Porcentaje de compras } \\
\text { realizadas }\end{array}$ & 20.00 & 10.00 & 25.00 & 45.00 & 100.00 \\
\hline & $\begin{array}{l}\text { Mz de tierra } \\
\text { compradas }\end{array}$ & 263 & 52 & 307 & 1394 & 2016 \\
\hline & $\begin{array}{l}\text { Porcentaje de tierra } \\
\text { comprado }\end{array}$ & 13.05 & 2.58 & 15.23 & 69.15 & 100.00 \\
\hline
\end{tabular}

Fuente: Elaboración propia en base a datos de encuesta.

El cuadro 7 muestra que los productores que suplen a la cadena de la leche fría pasteurizada son quienes principalmente han comprado más tierra, en comparación con aquellos productores que suplen canales alternativos de comercialización. En el caso de la tierra adyacente a la finca, el porcentaje más alto de productores que reporta este tipo de compra (20.69\%) se encuentra en el grupo de productores que vende a los acopios. Adicionalmente, estos productores son responsables de la mayor parte de las compras reportadas (34.09\%) así como del mayor porcentaje de tierra adquirida (68.17\%). Similarmente, también los productores que venden a los acopios reportan el mayor porcentaje de individuos que ha realizado compras (13.79\%), con la mayor proporción total de las compras (45\%) y el mayor porcentaje de tierra adquirida como nuevas fincas $(69.15 \%)$.

Estas tendencias en el mercado de tierra local confirman que los precios más altos y estables pagados por los acopios han alentado la compra de tierras, siendo aquellos productores capaces de vender a los acopios los que han comprado y acumulado tierra que está siendo destinada a la producción de más leche. La tierra en venta proviene de productores pequeños $\mathrm{y} / \mathrm{o}$ pobres quienes, probablemente, son incapaces de vender a la cadena de leche fría pasteurizada por diferentes restricciones. 
Esta falta de oportunidades económicas para un segmento de productores junto con la diferencia de precios de la tierra respecto a la frontera agrícola, ha alentado la venta de tierra y la migración hacia la misma, impulsando el avance de la frontera agrícola y la deforestación en Nicaragua.

\section{Conclusiones}

El modelo actual de producción de leche vinculado al boom de los productos lácteos se apoya en las ventajas que los productores grandes y/o capitalizados tienen para capturar los beneficios derivados de la cadena de la leche fría pasteurizada: precios más altos y estables durante el año. Adicionalmente, la producción ganadera lechera continua llevándose a cabo de manera extensiva a pesar del apoyo brindado por organismos de desarrollo e instituciones financiaras para promover la reconversión e intensificación de la producción. En este sentido, Matiguás y sus productores han logrado avances muy modestos. Bajo la lógica actual de producción, los productores han crecido y continúan creciendo bajo el modelo de adición de más tierra a la producción en lugar de adoptar prácticas de producción intensivas y amigables con el medio ambiente.

Al mismo tiempo los productores que han logrado conectarse y suplir la cadena de la leche fría pasteurizada han comprado y acumulado más tierra que está siendo orientada a la producción ganadera lechera, dada la disponibilidad de tierra en venta en el territorio, en vez de realizar inversiones en finca encaminadas a la intensificación de la producción. La tierra en venta proviene de (pequeños y medianos) productores que enfrentan diferentes restricciones (de tierra, ganado propio y crédito para inversiones en finca) que les impide vender a los centros de acopio y beneficiarse de los precios más altos y estables que éstos pagan. La falta de oportunidades económicas para este tipo de productores, junto con grandes diferencias de precios de tierra entre la vieja y la nueva frontera agrícola, ha alentado la venta de tierra en Matiguás y la migración interna hacia otros territorios al este del país, sin poder detener o reducir el avance de la frontera agrícola en Nicaragua.

\section{Referencias bibliográficas}

Barrios, C A. (2002). La ganadería como oportunidad de negocio para las microfinanzas: consideraciones generales sobre el funcionamiento técnico y los mercados de productos ganaderos. Managua: PROMIFIN COSUDE.

BCN. (2011). InformeAnual. On line available at http://www.bcn.gob.ni/ publicaciones/anual/memoria/Informe_anual_2011.pdf (last consulted: 17 November 2012).

Bebe, B.O., Udo, H.M.J., Rowlands, G.J. \& Thorpe, W., (2003). Smallholder dairy systems in the Kenya highlands: breed preferences and breeding practices. Livestock Production Science, 82, 117-127.

CENAGRO (2001). Censo Nacional Agropecuario, tercero. Managua, INIDE.

Collins, J. L., \& Painter, M., (1986). Settlement and Deforestation in Central America: A Discussion of Development Issues. Working Paper No. 31, Institute for 
Development Anthropology. New York: Clark University.

Dale, V. H. (1997). The relationship between land-use change and climate change. Ecological Applications, 7, 753-769.

Fearnside, P.M., (2005). Deforestation in Brazilian Amazonia: history, rates, and consequences. Conservation Biology, 19, 680-688.

Galetto, A. \& Berra, C. (2011). Dairy Development in Nicaragua and Farmer Cooperatives in the Matagalpa Region. Presented at International Food \& Agribusiness Management Association 21st. Annual World Symposium. Frankfurt, 20-23, (PAPER)

Geist, H.J. \& Lambin, E.F., (2002). Proximate Causes and Underlying Driving Forces of Tropical Deforestation.BioScience, 52 (2), 143-150.

Grau, H.R. \& Aide, T.M., (2008). Globalization and land use transitions in Latin American. Ecology and Society, 13, 16-28.

Griffith, K. and Zepeda, L. (1994). Farm level trade-offs of intensifying tropical milk production. EcologicalEconomics, 9, 121-133.

Holmann, F. (1999). Análisis ex-ante de nuevas alternativas forrajeras en fincas con ganado de doble propósito en Perú, Costa Rica y Nicaragua. PasturasTropicales , 21(2), 2-17.

Humphries, S. (1998). Milk cows, migrants, and land markets: unraveling the complexities of forest-to-pasture conversion in northern Honduras. Economic Development and Cultural Change, 47, 95-124.

Kaimowitz, D., \& A. Angelsen. (1998). Economic models of tropical deforestation: A review. Jakarta: Centre for International ForestryResearch.

Kaimowitz, D., (1996). El Avance de la Agricultura Sostenible en América Latina. Agroecologia y Desarrollo, 10,2-9.

MAGFOR. (2005). Programa de Reactivación Agropecuario Rural Sub-componente II-2: Apoyo a la Estrategia de Desarrollo de la Economía Rural. Managua: MAGFOR.

MAGFOR. (2008a). Subprograma de reconversión de la ganadería bovina en Nicaragua. On line available at http://www.magfor.gob.ni/prorural/programasnacionales/ perfilessub/ganaderia.pdf_(last consulted: 14 June 2011).

MAGFOR (2010). Sistema de Información Agropecuaria y Forestal; subsistema de inteligencia de precios y mercados agropecuarios. On line available at http:// www.magfor.gob.ni/sim/ (last consulted: 3 March 2013).

Maher, D., \& Schneider, R. (1994). Incentives for tropical deforestation: some examples from Latin America. In BROWN, K., PEARCE, D.W. (Eds.). The Causes of Tropical Deforestation: The Economic and Statistical Analysis of Factors Giving Rise to the Loss of Tropical Forests (159-171). London: UniversityCollege London Press.

Maldidier, C. (1993). Tendencias Actuales de la Frontera Agrícola en Nicaragua. Managua: Nitlapan-UCA.

Maldidier, C. \& Antillón, T. (1996). Deforestación y Frontera Agrícola en Nicaragua. En Frontera Agricola en Nicaragua. Managua: NitlapanUCA.

Mordt, M. (2002). Sustento y Sostenibilidad en la Frontera Agrícola: La Evolución de la Frontera en el Sudeste de Nicaragua. Managua: Nitlapán-UCA.

Nicholson, C. F., Blake, R. W. \& Lee. D. R. (1995). Livestock, deforestation, and policy making: intensification of cattle production systems in Central America 
Encuentro No. 104, 6-28, 2016

revisited. Journal of Dairy Science, 78, 719-734.

Perez, J F. (2011). Nicaragua: without structural changes there will be no sustainable reduction of rural poverty. Envio, 14-23.

Polvorosa, J. (2013). Opportunities and constraints from small and medium sized dairy farmers in the context of the booming dairy value chain in Nicaragua; case-study of Matiguás.Tesis Doctoral, Antwerp: UA Publications.

Sánchez, J. (2000). Utilización Eficiente de las Pasturas Tropicales en la Alimentación del Ganado Lechero. Presented at XI Seminario Manejo y Utilización de Pastos y Forrajes en Sistemas de Producción Animal. Centro de Investigaciones en Nutrición Animal. San José, 12-14 Abril.

Steinfeld, H., Gerber, P., Wassenaar, T., Castel, V., Rosales, M., \& DeHaan, C., (2006).Livestock's long shadow: environmental issues and options. Rome: Food and Agriculture Organization of the United Nations.

Tomich T.P., Van Noordwijk M., Vosti S. \& Witcover J. (1998). Agricultural development with rainforest conservation: Methods for seeking best bet alternatives to slash-and-burn, with applications to Brazil and Indonesia. Agricultural Economics, 19, 159-174.

White, D., Holmann, F., Fijusaka, S., Reategui, K., \& Lascano, C., (2001). Will intensifying pasture management in Latin America protect forests-or is it the other way round? In: A. Angelsen \& D. Kaimowitz (Eds.). Agricultural Technologies and Tropical Deforestation, (91-111). Wallingford: CABI Publishing.

Yamamoto, W., Dewi, I. A., \& Ibrahim, M. (2007).Effects of silvopastoral areas on milk production at dual-purpose cattle farms at the semi-humid old agricultural frontier in central Nicaragua. AgriculturalSystems, 94, 368-375. 